\title{
Mapping the Flow of Pediatric Trauma Patients Using Process Mining
}

Ashimiyu B. Durojaiye ${ }^{1,2}$ Nicolette M. McGeorge ${ }^{2}$ Lisa L. Puett ${ }^{3} \quad$ Dylan Stewart $^{4}$ James C. Fackler ${ }^{5}$ Peter L. T. Hoonakker ${ }^{6}$ Harold P. Lehmann ${ }^{1}$ Ayse P. Gurses ${ }^{1,2,7,8,9}$

\footnotetext{
${ }^{1}$ Division of Health Sciences Informatics, Johns Hopkins University School of Medicine, Baltimore, Maryland, United States

${ }^{2}$ Armstrong Institute Center for Health Care Human Factors, Johns Hopkins Medicine, Baltimore, Maryland, United States

${ }^{3}$ Department of Pediatric Nursing, Johns Hopkins Hospital, Baltimore, Maryland, United States

${ }^{4}$ Department of Pediatric Surgery, Johns Hopkins University School of Medicine, Baltimore, Maryland, United States

${ }^{5}$ Division of Pediatric Anesthesiology and Critical Care Medicine, Department of Pediatrics, Johns Hopkins University School of Medicine, Baltimore, Maryland, United States

${ }^{6}$ Center for Quality and Productivity Improvement, University of Wisconsin, Madison, Wisconsin, United States

${ }^{7}$ Department of Anesthesiology and Critical Care Medicine, Johns Hopkins University, Baltimore, Maryland, United States

8 Malone Center for Engineering in Healthcare, Johns Hopkins University School of Medicine, Baltimore, Maryland, United States 9 Department of Health Policy and Management, Bloomberg School of Public Health, Johns Hopkins University, Baltimore, Maryland, United States
}

Appl Clin Inform 2018;9:654-666.
Address for correspondence Ayse P. Gurses, PhD, MS, MPH, Department of Anesthesiology and Critical Care Medicine, Armstrong Institute Center for Health Care Human Factors, Johns Hopkins University, 750 East Pratt Street, 15th Floor, Baltimore, MD 21202, United States (e-mail: agurses1@jhmi.edu).

\section{Abstract \\ Keywords \\ - pediatric trauma \\ - process mining \\ - trauma registry \\ - patient pathway \\ - care transition \\ - conformance checking}

Background Inhospital pediatric trauma care typically spans multiple locations, which influences the use of resources, that could be improved by gaining a better understanding of the inhospital flow of patients and identifying opportunities for improvement.

Objectives To describe a process mining approach for mapping the inhospital flow of pediatric trauma patients, to identify and characterize the major patient pathways and care transitions, and to identify opportunities for patient flow and triage improvement. Methods From the trauma registry of a level I pediatric trauma center, data were extracted regarding the two highest trauma activation levels, Alpha $(n=228)$ and Bravo $(n=1,713)$. An event log was generated from the admission, discharge, and transfer data from which patient pathways and care transitions were identified and described. The Flexible Heuristics Miner algorithm was used to generate a process map for the cohort, and separate process maps for Alpha and Bravo encounters, which were assessed for conformance when fitness value was less than 0.950 , with the identification and comparison of conforming and nonconforming encounters.

Results The process map for the cohort was similar to a validated process map derived through qualitative methods. The process map for Bravo encounters had a relatively low fitness of 0.887 , and $96(5.6 \%)$ encounters were identified as nonconforming with characteristics comparable to Alpha encounters. In total, 28 patient pathways and 20 care transitions were identified. The top five patient pathways were received

March 1, 2018

accepted after revision

June 27, 2018 (c) 2018 Georg Thieme Verlag KG Stuttgart · New York
DOI https://doi.org/ 10.1055/s-0038-1668089. ISSN 1869-0327. 
traversed by $92.1 \%$ of patients, whereas the top five care transitions accounted for $87.5 \%$ of all care transitions. A larger-than-expected number of discharges from the pediatric intensive care unit (PICU) were identified, with $84.2 \%$ involving discharge to home without the need for home care services.

Conclusion Process mining was successfully applied to derive process maps from trauma registry data and to identify opportunities for trauma triage improvement and optimization of PICU use.

\section{Background and Significance}

Trauma is the leading cause of morbidity and mortality among children in the United States. ${ }^{1,2}$ Every year, approximately 9 million children are evaluated in emergency departments (ED) for traumatic injury, resulting in roughly 225,000 admissions, and approximately 10,000 deaths are recorded. $^{3}$ The annual direct economic cost of pediatric trauma is estimated to top US $\$ 50$ billion. ${ }^{4}$

Pediatric trauma patients often present with multiple injuries, requiring multidisciplinary care and coordination of diverse team members who work across time and space. ${ }^{4-6}$ Inhospital pediatric trauma care usually begins in the ED and, depending on injury severity, ends in discharge or leads to admission, which involves transitions and care in different areas of the hospital (e.g., operating room [OR], intensive care unit [ICU], or floor). ${ }^{4,7}$ The sequence of care transitions (or patient pathways) depends on the nature and severity of a patient's injury, and the sum of these pathways (or inhospital patient flow) is a measure of the efficiency of a hospital. ${ }^{9}$

To the best of our knowledge, there has been little research on the system-based assessment of patient flow through a pediatric trauma center. Most research has focused on improving adult patient flow through regional trauma networks ${ }^{8,10,11}$ and within and around the ED of a trauma center. ${ }^{12-14}$ However, such local optimization does not necessarily improve overall system performance due to hospital-wide interdependencies. ${ }^{15}$ Furthermore, certain trauma care transitions are associated with higher risks to patient safety, ${ }^{14,16}$ and with the current efforts to contain health care costs, ${ }^{17}$ it is important to minimize the number of care transitions and improve existing care transitions processes. To achieve this, it is necessary to gain a better understanding of patient flow through the trauma center.

\section{Objectives}

The objectives are to describe a process mining approach for mapping the hospital flow of pediatric trauma patients, to identify the most common patient pathways and care transitions, and to identify opportunities for patient flow and triage improvement.

\section{Methods}

\section{Study Setting}

The Johns Hopkins Children's Center is a level I pediatric trauma center in Baltimore, Maryland, United States. Every year, approximately 1,000 injured children are evaluated in the pediatric ED. Based on prehospital injury, anatomical, and physiological information, incoming patients are triaged to one of four trauma activation levels (Alpha, Bravo, Consult, and ED response) that are derivatives of the American College of Surgeons Committee on Trauma guidelines. ${ }^{18}$ An Alpha activation mobilized clinicians in the ED, the pediatric ICU (PICU), and the pediatric trauma service for children with severe and potentially life-threatening injuries most often destined for the PICU. Bravo activation mobilized clinicians in the ED and the trauma service for children with less critical injuries. Relatively stable patients activate a Consult for the pediatric trauma service, which include patient transfers from other facilities, whereas patients with minor injuries prompt an ED response. At any point during an evaluation, the trauma activation level can be changed if necessary.

\section{Data Source and Variables}

Data were obtained from the Pediatric Trauma Registry that is managed by the pediatric trauma program and has been operational since 1992. In July 2012, the registry transitioned to the state-mandated Maryland Trauma Registry (MTR) provided by Digital Innovations. The inclusion criteria and data fields are defined in the Maryland State Trauma Registry Data Dictionary for Pediatric Patients, ${ }^{19}$ which conforms to the National Trauma Data Standard (NTDS). ${ }^{20}$ Two full-time American Trauma Society trained registrars manage the registry. All data are manually and concurrently abstracted. Validation checks defined by the NTDS and the state, and in the MTR software, are run prior to commit. In addition, every month, at least $10 \%$ of new records are validated by reabstraction.

Our unit of analysis was the patient encounter, and multiple, independent encounters involving the same patient were treated as separate encounters. We included encounters managed between January 1, 2013, and December 31, 2017, with an Alpha or Bravo activation that involved none or one visit to the OR. We chose Alpha and Bravo activations because we sought to understand the flow of the sickest patients requiring multidisciplinary care. The following variables were extracted for each encounter:

- Patient encounter characteristics: medical record number, encounter number, date of birth, gender, origin of patient (scene of injury, transfer, or others), initial trauma activation (Alpha or Bravo), trauma activation change (upgrade, downgrade, or no change), injury type (blunt, penetrating, 
or others), mode of arrival (ground ambulance, helicopter, or others), Glasgow Coma Scale (GCS) score at ED arrival, Injury Severity Score (ISS), ED length of stay (LOS), PICU LOS, and hospital LOS.

- Admission, discharge, and transfer (ADT) data: timestamps at the minute level of granularity of ED arrival, ED departure (and ED disposition), first visit to the OR (and OR disposition), first visit to the PICU, first visit to the floor, and hospital discharge (and hospital disposition). Timestamps of visits to imaging locations were not collected as they do not constitute care transitions.

\section{Process Mining}

Process mining is a field of data science that "aims to discover, monitor, and improve real processes by extracting knowledge from event logs." ${ }^{21}$ It supports the analyses of various perspectives of processes, including control-flow analysis, which is the analysis of the sequence in which activities occur, and the perspective of interest in this study. The starting point for process mining is an event $\log ,{ }^{22}$ which contains a collection of events. Each event represents a welldefined activity (e.g., admission to the ED) in a given process (e.g., patient flow) and relates to a case (in our study, patient encounter). Each event is timestamped (e.g., patient arrived ED at 12/31/1990 16:40:00), allowing all events for a patient encounter to be ordered chronologically. An ordered sequence of events is called a trace (in our study, patient pathway), which represents an instance of the process. Many patient pathways can be combined using a variety of process discovery algorithms to derive a process model (in our study, a process map). ${ }^{22}$ The derivative patient pathways can be replayed on the process map to identify patient pathways that conform to the process map, and those that do not, in a process known as conformance checking. ${ }^{23,24}$

\section{Study Design}

We adapted and incorporated Lismont et al's ${ }^{25}$ process mining methodology, which uses an iterative approach to tackle the peculiarities of health care data. The overview of our study design is depicted in - Fig. 1. The following sections outline the implementation of the aspects of the study.

\section{Event Log Preparation}

We randomly assigned a case ID to each encounter and used date shifting ${ }^{26}$ to deidentify all timestamps to maintain the temporal sequence of events. The day of the week (weekday or weekend) and time of day (morning or night) at the start of encounter were also preserved. Missing or invalid timestamps were replaced with "not available." A valid timestamp for an inpatient location indicated admission of a patient at that location, and we created an event that was coded with the study-generated case ID, the inpatient admission location for the activity, and the timestamp. Generation of an event was contingent on ED disposition; records with missing ED disposition were excluded. Patients who were transferred to other hospitals were also excluded. When the ED disposition was to an inpatient location, the timestamps for all inpatient locations were processed. When ED disposition was discharge or death, records with valid inpatient timestamps were excluded. To capture the prehospital to ED care transition, which could not be naturally captured in our dataset, we created an "origin" event at the beginning of every encounter. For encounters that involved a visit to the OR, we also created an inpatient event from the OR disposition with the timestamp taken as 2 minutes after the patient was admitted to the OR. The goal was to capture where the patient was admitted after the OR, which could be a second admission to that location, since our registry only captured the first visits.

\section{Event Log Inspection}

Patient pathways were visually explored and suspected anomalies were identified and reviewed by examining the raw registry data. Anomalous patient pathways did not follow a logical sequence for trauma care (e.g., start of event was admission to a general care floor, care transition was to the same location). When the etiology could be determined, the patient pathways were fixed and retained, otherwise they were excluded.

\section{Clustering}

Encounters with exactly matching patient pathways were grouped together and patient pathway groups were numbered based on the frequency of occurrence. Each encounter

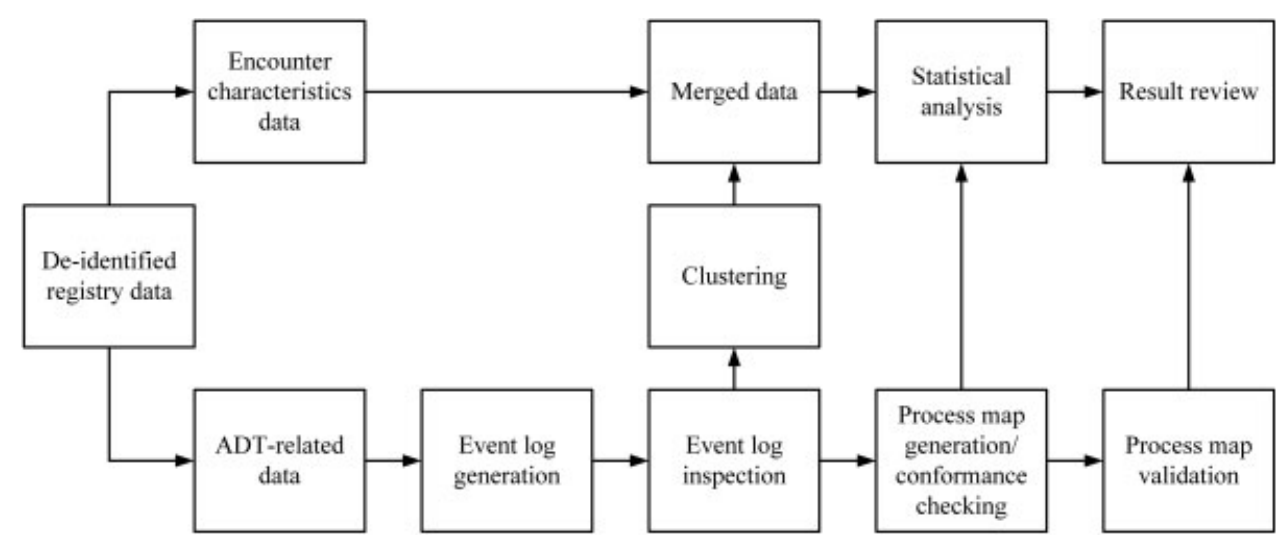

Fig. 1 Overview of the methodology used in this study. The process mining technique was applied to the admission, discharge, and transfer data. The resulting data were merged with the patient encounter characteristic data to provide clinical context for analysis. 
was assigned the unique number of the patient pathway group to which it belonged. Care transitions (defined as any two consecutive events in a patient pathway) were identified and numbered based on the frequency of occurrence. Each encounter was assigned the number(s) indicating the involved care transitions. Using the unique study-generated case ID as the merge key, we merged the resulting datasets with the patient encounter characteristics dataset to obtain the final dataset.

\section{Process Map Generation and Conformance Checking}

The event log was imported into ProM 6.6, which is an opensource platform that supports a wide variety of process mining algorithms and techniques, ${ }^{27}$ and was converted to the eXtensible Event Stream (XES) file format, which is the standard file format for event logs. ${ }^{28}$ The Flexible Heuristics Miner (FHM) algorithm was used to generate a process map for the entire cohort, and separate process maps for Alpha and Bravo encounters independently. The FHM algorithm was selected because it can handle lowly structured processes, noise (infrequent events), and loops, ${ }^{29}$ and it is regarded as one of the best process discovery algorithm for real-life data. ${ }^{30}$ The output of the FHM algorithm is a Heuristics Net that shows a "general" map of the process ${ }^{31}$ with noise silenced out.

Given the relatively low frequency of some events (e.g., death) and care transitions (e.g., OR to Died), the settings of the FHM algorithm were carefully adjusted to ensure that these events and transitions were not treated as noise while being careful not to allow too much noise, which defeated the purpose of using the algorithm. The "relative-to-best" threshold, which determines the amount of tolerable noise, ${ }^{29,31}$ was eventually set at $20 \%$ from the default of $5 \%$, whereas the "dependency threshold," which is the minimum threshold for accepting dependency (connections) between events, ${ }^{29,31}$ was eventually set at $75 \%$ from the default of $90 \%$. The quality of the derived process maps was evaluated by the fitness metric that is calculated as defined by Weijters et $\mathrm{al}^{31}$ and provided by the algorithm. This metric quantifies how well the resulting process map can reproduce the events from which it was derived. ${ }^{32}$ Fitness is the most important metric in evaluating process map quality. ${ }^{32}$ Fitness values range from 0 to 1 , and a value of $\geq 0.8$ is considered to be good for relatively wellstructured processes. $^{33}$

As there should be less variation (hence, less noise) in patient pathways for each trauma activation compared with the entire cohort, we expected the fitness value of the process map for each trauma activation to be higher than that of the entire cohort and closer to 1 . Consequently, when the fitness value of the process map for either trauma activation was less than 0.950 ( $5 \%$ noise allowed for normally occurring statistical exceptions), we performed conformance checking of the patient pathways of that trauma activation. To do so, the process map in question (a heuristic net) was converted to a Petri Net, which is a graphical, precise mathematical model of the process map. ${ }^{34}$ The events for that trauma activation were then replayed on the Petri Net using the "Replay Log on Petri Net for Conformance Check- ing" plugin in the default setting. This plugin employs a "costbased fitness" approach to measuring conformance. 35,36 Summary raw fitness costs were obtained, and patient pathways with raw fitness cost less than 2 standard deviation (SD) from the mean were identified and classified as nonconforming encounters.

\section{Process Map Validation}

A process map of prehospital care to hospital discharge was independently developed by reviewing the institutional pediatric trauma protocols and guidelines. This qualitative process map was iteratively refined as part of interviews with clinicians in the ED, PICU, and surgery service who were involved with pediatric trauma patients $(n=12)$ and finalized through member-checking interviews $(n=3)$ with subject matter experts on the research team. The process mining derived cohort process map was subsequently visually compared with the hospital phase of the qualitatively derived process map ( $\sim$ Appendix A).

\section{Statistical Analysis}

Descriptive analyses were performed for the demographic, injury, and admission characteristics of the patient encounters, patient pathways, and care transitions. We compared Alpha trauma encounters with Bravo trauma encounters, and conforming and nonconforming encounters of either trauma activation, when indicated. Wilcoxon's rank-sum and Pearson's chi-square tests were used to examine differences in interval and categorical variables, respectively; an $\alpha$ of $<0.05$ determined statistical significance. Analyses were performed in Stata $13 .^{37}$

\section{Results}

There were 1,985 encounters extracted from the trauma registry. A total of 465 (23.4\%) encounters had missing timestamps of the inpatient location they were admitted to from the ED, and these timestamps were replaced by the ED departure timestamps, which were the same as the timestamp of admission to the ED disposition in $61.8 \%$ of encounters without missing data. In total, 44 (2.2\%) encounters were excluded for ADT-related data quality issues, which included 22 (50\%) of 44 anomalous patient pathways that could not be salvaged ( - Fig. 2). The etiology of these data quality issues were identified as data entry errors (-Appendix B).

The final dataset contained 1,941 encounters: 228 (11.7\%) Alpha and 1713 (88.3\%) Bravo encounters. All clinical data fields in the final dataset had near perfect completeness values except ISS and mode of arrival with 68 (3.5\%) and 13 (0.7\%) missing values, respectively. Compared with Bravo encounters, Alpha encounters had a significantly lower median GCS (15 vs. 13; $p<0.001$ ), a higher median ISS ( 4 vs. $10 ; p<0.001$ ), a higher proportion of penetrating injuries ( 2.6 vs. $18.9 \% ; p<0.001$ ), higher proportion of PICU admissions ( 11.5 vs. $57.9 \% ; p<0.001$ ), longer median PICU LOS ( 1 vs. 3 days; $p<0.001$ ), and longer median hospital LOS ( 1 vs. 2.5 days; $p<0.001$ ) ( $>$ Table 1 ). 


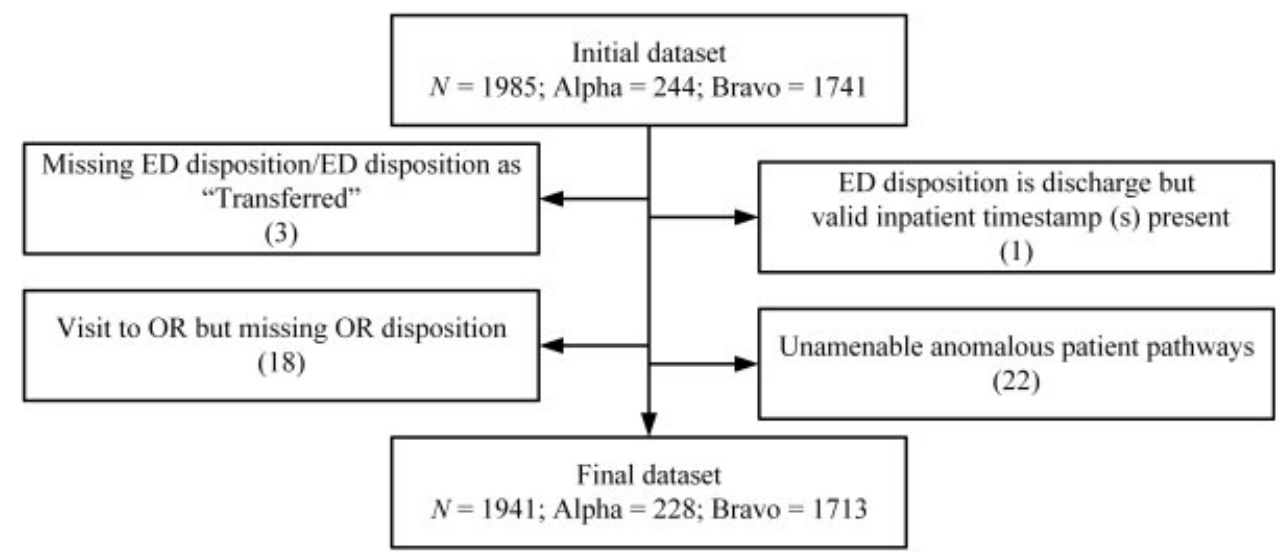

Fig. 2 Study flow diagram of included encounters and reasons for excluded encounters. ED, emergency department; OR, operating room.

\section{Process Maps}

The process map for the cohort had a fitness of 0.897 . Admitted patients could loop between the OR, PICU, and floor and could be discharged from the PICU, floor, or postanesthesia care unit (-Fig. 3). Notably, the transition from floor to PICU was not depicted. The process map for Alpha encounters had a fitness of 0.975 and featured a loop around the OR, PICU, and floor, whereas the process map for Bravo encounters had much less fitness of 0.886 and was unidirectional, involving mainly the floor (-Fig. 4). Conformance checking of Bravo encounters against the Bravo process map gave a summary raw fitness cost of $2.28 \pm 0.57$ SD (-Fig. 5). Ninety-six (5.6\%) encounters had a raw fitness cost of 1 out of 4 and were identified nonconforming. Compared with conforming encounters, nonconforming encounters had a significantly higher median ISS (4 vs. $10 ; p<0.001$ ), higher proportion of penetrating injuries ( 0 vs. $2.7 \%$; $p=0.003$ ), higher proportion of PICU admission ( 6.6 vs. 93.8\%; $p<0.001)$, and longer hospital LOS ( 1 vs. 2 days; $p<0.001$ ) ( - Table 2). They also tend to arrive less during the day shift ( 66.9 vs. $56.3 \% ; p<0.032$ ) and arrive as transfers ( 10.8 vs. $43.8 \%$; $p<0.001$ ).

\section{Patient Pathways}

Twenty-eight different patient pathways were identified. The top five patient pathways accounted for $92.1 \%$ of all encounters ( $\rightarrow$ Table 3 ). The most common patient pathway was origin $\rightarrow$ ED $\rightarrow$ discharge, which accounted for $45.2 \%$ of all encounters. Eight patient pathways were unique (had at most two occurrences) and accounted for $0.5 \%$ of all encounters. Four of these patient pathways ended in death (-Appendix C).

\section{Care Transitions}

Twenty different care transitions were identified. The top six transitions accounted for $87.5 \%$ of all transitions (-Table 4). The least common care transitions were OR to death and floor to PICU with four and five occurrences, respectively (-Appendix D). A total of 120 patients were discharged to home from the PICU, which was larger than expected. Further investigation revealed that Bravo encounters accounted for 190 (60.5\%) of 314 ED to PICU admissions and 92 (76.7\%) of PICU discharges to home, with 101 (84.2\%) discharged to home without the need for home care services. The median GCS on arrival and the median ISS of both care transitions were 15 and 9 to 10 , respectively.

\section{Discussion}

We used process mining on trauma registry data to map patient flow at a level I pediatric trauma center with the objective of exploring whether and where flow and triage could be improved. To our knowledge, this is the first time process mining has been used on trauma registry data. The process map for the cohort had a good fitness value, demonstrating that the process map could be replayed using the algorithm and dataset. Our process map was similar to the process map that we independently derived from qualitative methods at the same institution. This comparison suggests that our process mining approach was reliable and that our cohort process map has face validity. Moreover, our process map was similar to the process map that was derived at another pediatric trauma center using a mixed method approach $^{38}$; however, their process map included the floor to PICU care transition. This transition was not depicted in both process maps that we derived from process mining and qualitative method. This likely reflected the infrequent nature (only 5 instances in the dataset) of the floor to PICU care transition at our institution and their treatment as noise by the Heuristics Miner algorithm since there were only a few instances relative to other transitions around the PICU and floor.

The large number of patient pathways revealed by this control-flow analysis illustrated the complexity of pediatric trauma care at the study site, and additional complexity may be revealed when other perspectives are considered. Although a patient pathway depends on their needs, the myriad of pathways may increase the complexity of coordinating care for patients. Gaps in coordinating postresuscitation trauma care, especially when patients transitioned between teams and care locations, have been described. ${ }^{39}$ Thus, it is important 
Table 1 Encounter characteristics by trauma activation groups

\begin{tabular}{|c|c|c|c|}
\hline Variable & Alpha, $n=228$ & Bravo, $n=1,713$ & $\begin{array}{l}\text { Alpha vs. Bravo, } \\
p \text {-Value }\end{array}$ \\
\hline Age, years, median (IQR) & $7(2-12)$ & $8(3-12)$ & 0.501 \\
\hline Male gender, $n(\%)$ & $156(68.4)$ & $1,117(65.2)$ & 0.337 \\
\hline Weekday arrivals, $n$ (\%) & $155(68)$ & $1,200(70.1)$ & 0.522 \\
\hline Day shift arrivals, $n(\%)$ & $153(67.1)$ & $1,136(66.3)$ & 0.813 \\
\hline \multicolumn{4}{|l|}{ Origin, $n(\%)$} \\
\hline Scene of injury & $200(87.7)$ & $1,400(86.4)$ & \multirow[t]{3}{*}{0.401} \\
\hline Transfer & $24(10.5)$ & $216(12.6)$ & \\
\hline Others & $4(1.8)$ & $17(1)$ & \\
\hline \multicolumn{4}{|l|}{ Mode of arrival, $n(\%)$} \\
\hline Ground ambulance & $167(73.3)$ & $1,248(72.9)$ & \multirow[t]{3}{*}{0.675} \\
\hline Helicopter & $56(2.6)$ & $409(23.9)$ & \\
\hline Others & $5(2.2)$ & $56(3.3)$ & \\
\hline \multicolumn{4}{|l|}{ Injury type, $n(\%)^{a}$} \\
\hline Blunt & $172(75.4)$ & $1,626(94.9)$ & \multirow[t]{3}{*}{$<0.001$} \\
\hline Penetrating & $43(18.9)$ & $44(2.6)$ & \\
\hline Others & $13(5.7)$ & $43(2.5)$ & \\
\hline GCS at arrival, median (IQR) & $13(6-15)$ & $15(15-15)$ & $<0.001$ \\
\hline ISS, median (IQR) ${ }^{\mathrm{a}}$ & $10(4-17)$ & $4(1-9)$ & $<0.001$ \\
\hline PICU admission, $n(\%)^{a}$ & $132(57.9)$ & $197(11.5)$ & $<0.001$ \\
\hline PICU LOS, median (IQR) ${ }^{\mathrm{a}}$ & $3(1-5)$ & $1(1-2)$ & $<0.001$ \\
\hline Hospital LOS & $2.5(1-6)$ & $1(1-1)$ & $<0.001$ \\
\hline
\end{tabular}

Abbreviations: GCS, Glasgow Coma Scale; IQR, interquartile range; ISS, Injury Severity Score; LOS, length of stay; PICU, pediatric intensive care unit. ${ }^{\text {a }}$ Statistically significant at alpha $<0.05$.

to focus research on improving this aspect of trauma care, such as redesigning the trauma work systems ${ }^{40}$ to minimize (if possible) and improve care transition processes. For example, health information technology (HIT) could be designed to support teamwork and coordination across settings; current HIT solutions are far from optimal. ${ }^{41,42}$

The median ISS of patients arriving at the ED was 4, which is in line with national statistics ${ }^{43}$ and an observed trend of overtriaging to level I trauma centers. ${ }^{44}$ While current triage philosophy prioritizes minimizing undertriage over overtriage from the field to the trauma center, ${ }^{18}$ it is the responsibility of the trauma center to ensure optimal use of resources. ${ }^{45}$ Our findings suggest two opportunities for triage improvement at our center. First, mildly injured children most often activated a Bravo response, which was meant for more acute injuries. For example, looking at the

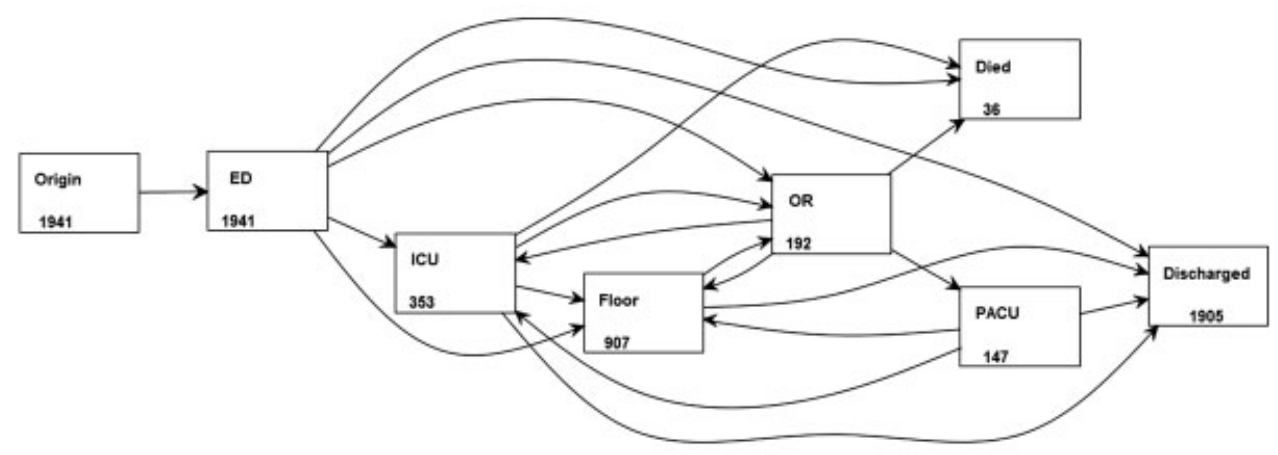

Fig. 3 The process map (fitness $=0.897$ ) of the cohort showing the flow of patients from arrival to discharge. The numbers in the boxes are the total number of admissions in that inpatient location. ED, emergency department (pediatric ED in this study); ICU, intensive care unit (pediatric ICU in this study); OR, operating room; PACU, postanesthesia care unit. 


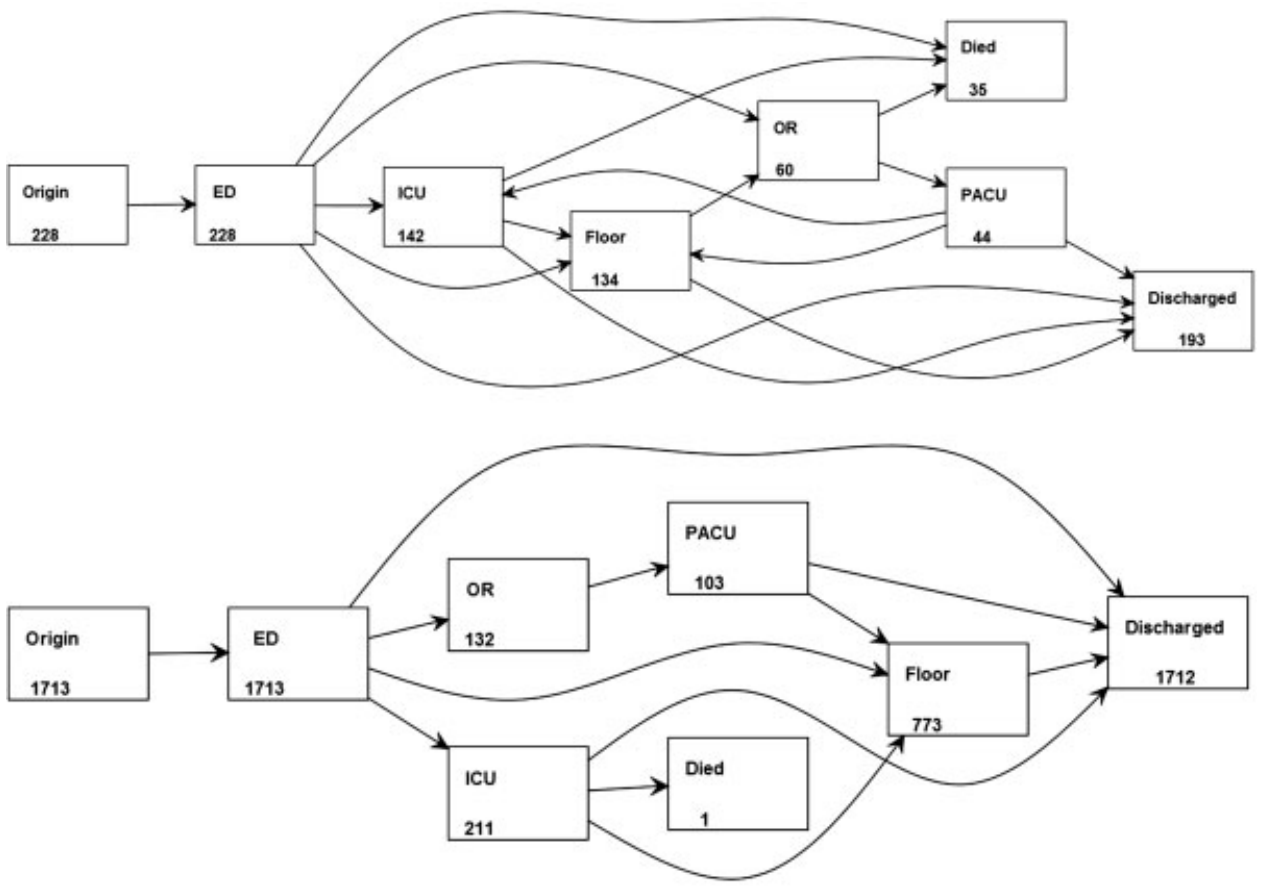

Fig. 4 The process map for the Alpha encounter (top, fitness value $=0.975$ ) and Bravo encounter (bottom, fitness value $=0.886$ ) cohort showing the flow of patients from arrival to discharge. The numbers in the boxes are the total number of admissions in that inpatient location. ED, emergency department (pediatric ED in this study); ICU, intensive care unit (pediatric ICU in this study); OR, operating room; PACU, postanesthesia care unit.

encounters that were discharged from the ED, the median age was 8 years, median ISS was 2, and median GCS was 15 . These characteristics suggested that these patients had minor injuries to a single body region and could possibly have been triaged to a lower trauma activation, such as a Consult. Overtriage disrupts and delays the provision of care to other nontrauma patients, which may have tangible and intangible costs. ${ }^{46}$ Second, $5.6 \%$ of Bravo encounters were shown to be strikingly different from other Bravo encounters but comparable with Alpha encounters, which suggested possible undertriage. Undertriage poses threat to patient safety and quality as it prevents or delays access to required care, which can result in less optimal outcomes. ${ }^{18}$ Although there is no reference target for hospital undertriage, the nonconforming Bravo encounters may benefit from an in-

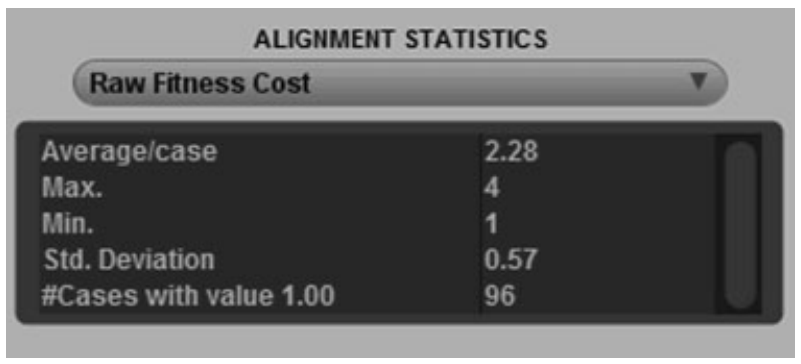

Fig. 5 Screenshot showing the summary raw fitness cost statistics obtained from conformance checking of Bravo encounters against the Bravo process map. Max., maximum; Min., minimum; Std; standard deviation. depth review to see if there were opportunities for triage improvement. Determining appropriate triage is difficult, and there is a significant variation in triage criteria used across pediatric trauma centers. ${ }^{47}$ At the study site, the current triage criteria could be refined by taking a closer look at Bravo encounters.

We also identified an opportunity for improvement in patient flow. Although Alpha encounters were more likely to involve PICU admission, our findings revealed that more Bravo encounters were actually admitted and discharged to home from the PICU. This pathway trend suggests potential overuse of the PICU. When discussed with the pediatric trauma program leaders at the study site, they suspected that this finding was most likely due to patients with isolated closed head injuries with skull fractures, intracranial hemorrhage, or both that require frequent neurologic checks, which was only available in the PICU. Our findings are similar to Fenton et al, ${ }^{48}$ who reported that $36 \%$ of ED to PICU admissions at their institution over a 13-year period were preventable and mostly involved isolated head injury cases. They attributed overuse to the lack of protocols to define criteria for PICU care creating reliance on the provider's experience, provider preference to err on the side of caution, and parental concern. ${ }^{48}$ Additional possible causes we elicited from the pediatric trauma program leaders and senior clinicians were a nonrefusal policy in the PICU; general care floor unit operating at capacity, diverting stable children to the PICU, and holding those ready for transition to the floor in the PICU; and lack of a formal intermediate care unit (IMCU) to manage patients who require frequent observations but 
Table 2 Comparison of Bravo process map conforming and nonconforming Bravo encounters ${ }^{\mathrm{a}}$

\begin{tabular}{|c|c|c|c|}
\hline Variable & $\begin{array}{l}\text { Conforming } \\
\text { Encounters, } \\
n=1,617\end{array}$ & $\begin{array}{l}\text { Nonconforming } \\
\text { Encounters, } \\
n=96\end{array}$ & $\begin{array}{l}\text { Conforming vs. } \\
\text { Nonconforming, } p \text {-Value }\end{array}$ \\
\hline Age, y, median (IQR) & $8(3-12)$ & $6(1.5-12)$ & 0.051 \\
\hline Male gender, $n(\%)$ & $1,052(65.1)$ & $65(67.7)$ & 0.596 \\
\hline Weekday arrivals, $n$ (\%) & $1,136(70.3)$ & $64(66.7)$ & 0.456 \\
\hline Day shift arrivals, $n(\%)^{\mathrm{a}}$ & $1,082(66.9)$ & $54(56.3)$ & 0.032 \\
\hline \multicolumn{4}{|l|}{ Origin, $n(\%)^{a}$} \\
\hline Scene of injury & $1,426(88.2)$ & $54(56.2)$ & \multirow[t]{3}{*}{$<0.001$} \\
\hline Transfer & $174(10.8)$ & $42(43.8)$ & \\
\hline Others & $17(1)$ & $0(0)$ & \\
\hline \multicolumn{4}{|l|}{ Mode of arrival, $n(\%)^{a}$} \\
\hline Ground ambulance & $1,208(74.7)$ & $40(41.7)$ & \multirow[t]{3}{*}{$<0.001$} \\
\hline Helicopter & $357(22.1)$ & $52(54.2)$ & \\
\hline Others & $52(3.2)$ & $4(4.2)$ & \\
\hline \multicolumn{4}{|l|}{ Injury type, $n(\%)^{a}$} \\
\hline Blunt & $1,537(95.1)$ & $89(92.7)$ & \multirow[t]{3}{*}{0.003} \\
\hline Penetrating & $44(2.7)$ & $0(0)$ & \\
\hline Others & $36(2.2)$ & $7(7.3)$ & \\
\hline GCS at arrival, median (IQR) & $15(15-15)$ & $15(14-15)$ & $<0.001$ \\
\hline ISS, median (IQR) & $4(1-6)$ & $9(5-14)$ & $<0.001$ \\
\hline ICU admission, $n(\%)^{\text {a }}$ & $107(6.6)$ & $90(93.8)$ & $<0.001$ \\
\hline ICU LOS, median (IQR) ${ }^{\mathrm{a}}$ & $1(1-2)$ & $1(1-2)$ & $<0.001$ \\
\hline Hospital LOS & $1(1-1)$ & $2(1-4)$ & $<0.001$ \\
\hline
\end{tabular}

Abbreviations: GCS, Glasgow Coma Scale; ICU, intensive care unit; ISS, Injury Severity Score; IQR, interquartile range; LOS, length of stay; PICU, pediatric intensive care unit.

${ }^{a}$ Statistically significant at alpha $<0.05$.

do not need the intense care provided in PICU. Given the high cost of care in PICU, further research is needed to better understand these causes and to find ways of optimizing the use of the PICU, which may include establishing formal requirements for PICU admission and building a formal IMCU capacity to cater to patients requiring frequent observations. For example, real-time decision support tools, such as that developed by Goldstein et $\mathrm{al}^{49}$ can be used to aid in the determination of the need for PICU admission.
The process mining methodology described in this paper has important implications for trauma centers. Trauma program directors can use process mining to obtain a better view of how patients are actually moving through their center. When trauma providers are in the midst of trauma care, there is a level of urgency, and their focus is on treating the patient no matter the pathway. Process maps illustrate the pathways, highlight patterns, and link them to contributing factors such as patient acuity. Longitudinal comparisons

Table 3 Top 5 Inhospital patient pathways $(N=1,941)$

\begin{tabular}{|l|l|l|l|l|l|l|}
\hline Rank & Patient pathways & $\begin{array}{l}\text { Frequency, } \\
n(\%)\end{array}$ & $\begin{array}{l}\text { Median } \\
\text { age (y) }\end{array}$ & $\begin{array}{l}\text { Median GCS } \\
\text { at arrival }\end{array}$ & $\begin{array}{l}\text { Median } \\
\text { ISS }\end{array}$ & $\begin{array}{l}\text { Median } \\
\text { hospital } \\
\text { LOS (d) }\end{array}$ \\
\hline 1 & Origin $\rightarrow$ ED $\rightarrow$ discharge & $877(45.2)$ & 8 & 15 & 2 & 1 \\
\hline 2 & Origin $\rightarrow$ ED $\rightarrow$ floor $\rightarrow$ Discharge & $582(30)$ & 7 & 15 & 5 & 1 \\
\hline 3 & Origin $\rightarrow$ ED $\rightarrow$ PICU $\rightarrow$ floor $\rightarrow$ discharge & $149(7.7)$ & 5 & 14 & 10 & 4 \\
\hline 4 & Origin $\rightarrow$ ED $\rightarrow$ PICU $\rightarrow$ discharge & $109(5.6)$ & 6 & 15 & 9 & 2 \\
\hline 5 & Origin $\rightarrow$ ED $\rightarrow$ floor $\rightarrow$ OR $\rightarrow$ PACU $\rightarrow$ discharge & $70(3.6)$ & 10 & 15 & 9 & 2.5 \\
\hline
\end{tabular}

Abbreviations: ED, emergency department; GCS, Glasgow Coma Scale; ISS, Injury Severity Score; LOS, length of stay; OR, operating room; PACU, postanesthesia care unit; PICU, pediatric intensive care unit. 
Table 4 Top six care transitions for pediatric trauma patient pathways $(N=5,481)$

\begin{tabular}{|l|l|l|l|l|l|l|}
\hline Rank & Care transition & $\begin{array}{l}\text { Frequency, } \\
\boldsymbol{n}(\%)\end{array}$ & $\begin{array}{l}\text { Median } \\
\text { age }(\mathrm{y})\end{array}$ & $\begin{array}{l}\text { Median GCS } \\
\text { at arrival }\end{array}$ & $\begin{array}{l}\text { Median } \\
\text { ISS }\end{array}$ & $\begin{array}{l}\text { Median hospital } \\
\text { LOS (d) }\end{array}$ \\
\hline 1 & Origin $\rightarrow$ ED & $1,941(35.4)$ & 8 & 15 & 4 & 1 \\
\hline 2 & ED $\rightarrow$ discharged & $877(16)$ & 8 & 15 & 2 & 1 \\
\hline 3 & Floor $\rightarrow$ discharged & $815(14.9)$ & 7 & 15 & 5 & 1 \\
\hline 4 & ED $\rightarrow$ floor & $666(12.2)$ & 8 & 15 & 5 & 1 \\
\hline 5 & ED $\rightarrow$ PICU & $314(5.7)$ & 6 & 15 & 10 & 3 \\
\hline 6 & PICU $\rightarrow$ floor & $180(3.3)$ & 5 & 14 & 10 & 4 \\
\hline
\end{tabular}

Abbreviations: ED, emergency department; GCS, Glasgow Coma Scale; ISS, Injury Severity Score; LOS, length of stay; PICU, pediatric intensive care unit.

of process maps and patient pathway patterns could help trauma centers identify any unexpected changes in patient flow and fix or mitigate problems. Since many trauma centers use the same state-mandated data fields in their trauma registries, this methodology can be applied at the state level for cross-organization process comparisons and benchmarking. ${ }^{50,51}$ This methodology can also be extended to support visualizations, such as that described by Chazard and Beuscart ${ }^{52}$ and visual analytics, ${ }^{53,54}$ which can be used by trauma program directors for administrative and quality improvement purposes. For example, process maps could facilitate discussions in mortality and morbidity rounds and quality improvement meetings.

Our study has some limitations. The trauma registry only captured the first visit to the various inpatient locations, and patients could be admitted to an inpatient location several times, especially encounters that involved multiple visits to the OR. Although we excluded encounters that involved more than one OR visit and examined OR dispositions to reveal subsequent admissions to various inpatient locations, it is possible that we did not capture all visits to an inpatient location and that some patient pathways may differ. Furthermore, the exclusion of encounters involving multiple visits to the OR means that not all existing patient pathways were identified. It is possible that the excluded encounters experienced unique and more diverse pathways. However, given the relatively small size of encounters with multiple visits to the $\mathrm{OR}(N=61$; Alpha $=27$; Bravo $=34)$, the unidentified patient pathways likely did not significantly impact the proof of method concept presented here or our general conclusions.

Furthermore, we generated events based on the assumption that the presence of a valid readable timestamp for an inpatient location represented a visit to that inpatient location. This assumption implicitly equates missing or invalid readable timestamps with a visit that did not happen, which is not necessarily true. Some visits may have happened but were not recorded or were not validly captured in the trauma registry. In addition, we were able to identify and fix inaccurate data points only when they resulted in anomalous pathways. For these two reasons, it is possible that some of the patient pathways are not truly accurate.

\section{Conclusion}

We employed a process mining approach to derive process maps using pediatric trauma registry data. We identified 28 patient pathways and 20 care transitions and opportunities for improving triage and optimizing the use of PICU. Future studies will explore the costs and mortality differences among the identified patient pathways.

\section{Clinical Relevance Statement}

One way of optimizing the use of trauma center resources is to gain a better understanding of patient flow and to improve hospital triage criteria. The process mining methodology we described can assist trauma centers in identifying opportunities for such improvements.

\section{Multiple Choice Question}

What kind of data are central to the application of process mining?
a. Location data
b. Timestamp data
c. Demographic data
d. Provider data

Correct Answer: The correct answer is option b, timestamp data. Process mining depends on timestamps data. Timestamp data can be sourced from different databases and processed into an event log, the starting point of process mining.

\section{Protection of Human and Animal Subjects}

This study was performed in compliance with the World Medical Association Declaration of Helsinki on Ethical Principles for Medical Research Involving Human Subjects and was reviewed by Johns Hopkins Medicine Institutional Review Board.

\section{Funding}

This study was supported by the Agency for HealthCare Research and Quality (R01HS023837; PI: Gurses). The study sponsor was not involved in the study design, the 
writing, and the decision to submit the manuscript for publication.

\section{Conflict of Interest}

Dylan Stewart and James Fackler are paid subject matter experts. The other authors do not report any conflict of interest.

\section{Acknowledgments}

We wish to thank Christine G. Holzmueller, BLA, for her thoughtful review and edits to the manuscript; she is a biomedical writer and editor at the Armstrong Institute for Patient Safety and Quality.

\section{References}

1 Centers for Disease Control and Prevention, National Center for Injury Prevention and Control. Web-based Injury Statistics Query and Reporting System (WISQARS). Fatal Injury Data. 2016. Available at: https://www.cdc.gov/injury/wisqars/fatal.html. Accessed March 1, 2018

2 Centers for Disease Control and Prevention, National Center for Injury Prevention and Control. Web-based Injury Statistics Query and Reporting System (WISQARS). Nonfatal Injury Data. 2016. Available at: https://www.cdc.gov/injury/wisqars/nonfatal.html Accessed March 1, 2018

3 Centers for Disease Control and Prevention, National Center for Injury Prevention and Control. National Action Plan for Child Injury Prevention. Atlanta, GA: CDC, NCIPC. 2012. Available at: https://www.cdc.gov/safechild/pdf/national_action_plan_for_child_injury_prevention.pdf Accessed March 1, 2018

4 Committee on Pediatric Emergency Medicine, Council on Injury; Violence, and Poison Prevention, Section on Critical Care, Section on Orthopaedics, Section on Surgery, Section on Transport Medicine, Pediatric Trauma Society, and Society of Trauma Nurses Pediatric Committee. Management of pediatric trauma. Pediatrics 2016;138(02):e20161569

5 Sharar SR. The ongoing and worldwide challenge of pediatric trauma. Int J Crit Illn Inj Sci 2012;2(03):111-113

6 McFadyen JG, Ramaiah R, Bhananker SM. Initial assessment and management of pediatric trauma patients. Int J Crit Illn Inj Sci 2012;2(03):121-127

7 American Academy of Pediatrics Committee on Pediatric Emergency Medicine; American College of Emergency physicians Pediatric Emergency Medicine Committee; Emergency Nurses Association Pediatric Committee. Handoffs: transitions of care for children in the emergency department. Pediatrics 2016;138(05):e20162680

8 Evans DC, Andrusiek DL, Sobolev B. Process mapping of a regional trauma system. In: Hall R, ed. Patient Flow: Reducing Delay in Healthcare Delivery. Boston, MA: Springer; 2013:311-332

9 Hall R, Belson D, Murali P, Dessouky M. Modeling patient flows through the health care system. In: Hall R, ed. Patient Flow: Reducing Delay in Healthcare Delivery. Boston, MA: Springer; 2013: 3-42

10 Ruchholtz S, Lewan U, Debus F, Mand C, Siebert H, Kühne CA. TraumaNetzwerk DGU(®): optimizing patient flow and management. Injury 2014;45(Suppl 3):S89-S92

11 Hipps D, Jameson S, Murty A, et al. The effect of introducing a Trauma Network on patient flow, hospital finances and trainee operating. Injury 2015;46(02):195-200

12 Oredsson S, Jonsson H, Rognes J, et al. A systematic review of triagerelated interventions to improve patient flow in emergency departments. Scand J Trauma Resusc Emerg Med 2011;19:43

13 Sayah A, Rogers L, Devarajan K, Kingsley-Rocker L, Lobon LF. Minimizing ED waiting times and improving patient flow and experience of care. Emerg Med Int 2014;2014:981472
14 Catchpole KR, Gangi A, Blocker RC, et al. Flow disruptions in trauma care handoffs. J Surg Res 2013;184(01):586-591

15 Kolker A. Interdependency of hospital departments and hospitalwide patient flows. In: Hall R, ed. Patient Flow: Reducing Delay in Healthcare Delivery. Boston, MA: Springer; 2013:43-63

16 Zakrison TL, Rosenbloom B, McFarlan A, et al. Lost information during the handover of critically injured trauma patients: a mixed-methods study. BMJ Qual Saf 2016;25(12):929-936

17 Mann NC, Mackenzie E, Teitelbaum SD, Wright D, Anderson C. Trauma system structure and viability in the current healthcare environment: a state-by-state assessment. J Trauma 2005;58(01): 136-147

18 American College of Surgeons Committee on Trauma. Resources for Optimal Care of the Injured Patient. Chicago, IL: American College of Surgeons; 2014

19 Maryland Institute for Emergency Medical Services Systems. Maryland State Trauma Registry Data Dictionary for Pediatric Patients. 2014. Available at: https://www.miemss.org/home/Portals/0/Docs/OtherPDFs/Web-registry-data-dictionary-pediatric. pdf?ver=2016-03-10-140444-350. Accessed March 1, 2018

20 American College of Surgeons, Committee on Trauma. National Trauma Data Standard Data Dictionary, 2017 Admissions. Available at: https://www.facs.org/ /media/files/quality\%20programs/trauma/ntdb/ntds/data\%20dictionaries/ntdsdatadictionary2017admissions.ashx. Accessed August 16, 2017

21 van der Aalst W. Process Mining: The missing link. Heidelberg: Springer; 2016:25-52

22 van der Aalst W. Process Mining: Data Science in Action. Heidelberg: Springer-Verlag; 2016

23 Rozinat A, van der Aalst WMP. Conformance checking of processes based on monitoring real behavior. Inf Syst 2008; 33:64-95

24 Rozinat A, Van der Aalst WM. Conformance testing: Measuring the fit and appropriateness of event logs and process models. International Conference on Business Process Management. Heidelberg: Springer; 2005:163-176

25 Lismont J, Janssens AS, Odnoletkova I, Vanden Broucke S, Caron F, Vanthienen J. A guide for the application of analytics on healthcare processes: a dynamic view on patient pathways. Comput Biol Med 2016;77:125-134

26 Liu J, Erdal S, Silvey SA, et al. Toward a fully de-identified biomedical information warehouse. AMIA Annu Symp Proc 2009;2009:370-374

27 Verbeek H, Buijs J, Van Dongen B, van der Aalst WMP. Prom 6: the process mining toolkit. In: Proceedings of BPM Demonstration Track. 2010;615:34-39

28 Paster F, Helm E. From IHE audit trails to XES event logs facilitating process mining. Stud Health Technol Inform 2015; 210:40-44

29 Weijters A, Ribeiro J. Flexible heuristics miner (FHM). In: Proceedings of the IEEE Symposium on Computational Intelligence and Data Mining CIDM. 2011:310-317

30 De Weerdt J, De Backer M, Vanthienen J, Baesens B. A multidimensional quality assessment of state-of-the-art process discovery algorithms using real-life event logs. Inf Syst 2012; 37:654-676

31 Weijters A, van der Aalst WMP, De Medeiros AA. Process mining with the heuristics miner-algorithm. Technische Universiteit Eindhoven. Tech Rep WP 2006;166:1-34

32 Buijs JC, Van Dongen BF, van der Aalst WMP. On the role of fitness, precision, generalization and simplicity in process discovery. OTM 2012;7565:305-322

33 van der Aalst WMP. Process mining: discovering and improving Spaghetti and Lasagna processes. In: Proceedings of the IEEE Symposium on Computational Intelligence and Data Mining CIDM. 2011:1-7

34 Desel J, Juhás G. "What Is a Petri Net?" Informal Answers for the Informed Reader In: Ehrig H, Padberg J, Juhás G, Rozenberg G, eds. 
Unifying Petri Nets. Lecture Notes in Computer Science. Vol. 2128. Berlin, Heidelberg: Springer; 2001:1-25

35 Adriansyah A, van Dongen BF, van der Aalst WM. Conformance checking using cost-based fitness analysis. In: Enterprise Distributed Object Computing Conference (EDOC), 15th IEEE International. IEEE;2011:55-64

36 van der Aalst WMP, Adriansyah A, van Dongen B. Replaying history on process models for conformance checking and performance analysis. Wiley Interdiscip Rev Data Min Knowl Discov 2012;2:182-192

37 StataCorp. 2013Stata Statistical Software: Release 13. College Station, TX: StataCorp LP

38 Wooldridge A, Carayon P, Hoonakker P, et al. Understanding Team Complexity in Pediatric Trauma Care [Abstract]. Paper presented at the Human Factors and Ergonomics in Healthcare, New Orleans, LA, March 5-8, 2017

39 Curtis K, Foster K, Mitchell R, Van C. How is care provided for patients with paediatric trauma and their families in Australia? A mixed-method study. J Paediatr Child Health 2016;52(08): 832-836

40 Carayon P, Schoofs Hundt A, Karsh BT, et al. Work system design for patient safety: the SEIPS model. Qual Saf Health Care 2006;15 (Suppl 1):i50-i58

41 Samal L, Dykes PC, Greenberg J, et al. The current capabilities of health information technology to support care transitions. AMIA Annu Symp Proc 2013;2013:1231

42 Institute of Medicine. Health IT and Patient Safety: Building Safer Systems for Better Care. Washington, DC: The National Academies Press; 2012

43 American College of Surgeons. National Trauma Data Bank 2016: Pediatric Annual Report. Available at: https://www.facs.org/ $\sim /$ media/files/quality\%20programs/trauma/ntdb/ntdb\%20pediatric \%20annual\%20report\%202016.ashx. Accessed October 24, 2017

44 Newgard CD, Staudenmayer K, Hsia RY, et al. The cost of overtriage: more than one-third of low-risk injured patients were taken to major trauma centers. Health Aff (Millwood) 2013;32 (09):1591-1599
45 Kouzminova N, Shatney C, Palm E, McCullough M, Sherck J. The efficacy of a two-tiered trauma activation system at a level I trauma center. J Trauma 2009;67(04):829-833

46 Dowd MD, McAneney C, Lacher M, Ruddy RM. Maximizing the sensitivity and specificity of pediatric trauma team activation criteria. Acad Emerg Med 2000;7(10):1119-1125

47 Drendel AL, Gray MP, Lerner EB. A systematic review of hospital trauma team activation criteria for children. Pediatr Emerg Care 2017. Doi: $10.1097 /$ PEC.0000000000001256

48 Fenton SJ, Campbell SJ, Stevens AM, Zhang C, Presson AP, Lee JH. Preventable pediatric intensive care unit admissions over a 13year period at a level 1 pediatric trauma center. J Pediatr Surg 2016;51(10):1688-1692

49 Goldstein SD, Cerullo M, Noje C, et al. Implementation of a Smartphone-based Decision Tool to Determine Level of Care for Pediatric Head Trauma [Abstract]. Paper presented at the 2017 4th Annual Meeting of the Pediatric Trauma Society, November 24, 2017, Charleston, SC

50 Suriadi S, Mans R, Wynn M, Partington A, Karnon J. Measuring patient flow variations: a cross-organisational process mining approach. In: Ouyang C, Jung JY (eds) Asia Pacific Business Process Management. AP-BPM 2014. Lecture Notes in Business Information Processing, vol 181. Cham: Springer

51 Partington A, Wynn M, Suriadi S, Ouyang C, Karnon J. Process mining for clinical processes: a comparative analysis of four Australian hospitals. ACM Trans Manag Inf Syst 2015;5:19

52 Chazard E, Beuscart R. Graphical representation of the comprehensive patient flow through the hospital. AMIA Annu Symp Proc 2007;2007:110-114

53 Kumar V, Park H, Basole RC, Braunstein M, Kahng M, Chau DH, et al. Exploring clinical care processes using visual and data analytics: challenges and opportunities. In Proceedings of the 20th ACM SIGKDD conference on knowledge discovery and data mining workshop on data science for social good. 2014

54 van der Aalst WMP, de Leoni M, ter Hofstede AH. Process mining and visual analytics: Breathing life into business process models. BPM Center Report BPM-11-15, BPMcenter.org 17 2011:699-730 


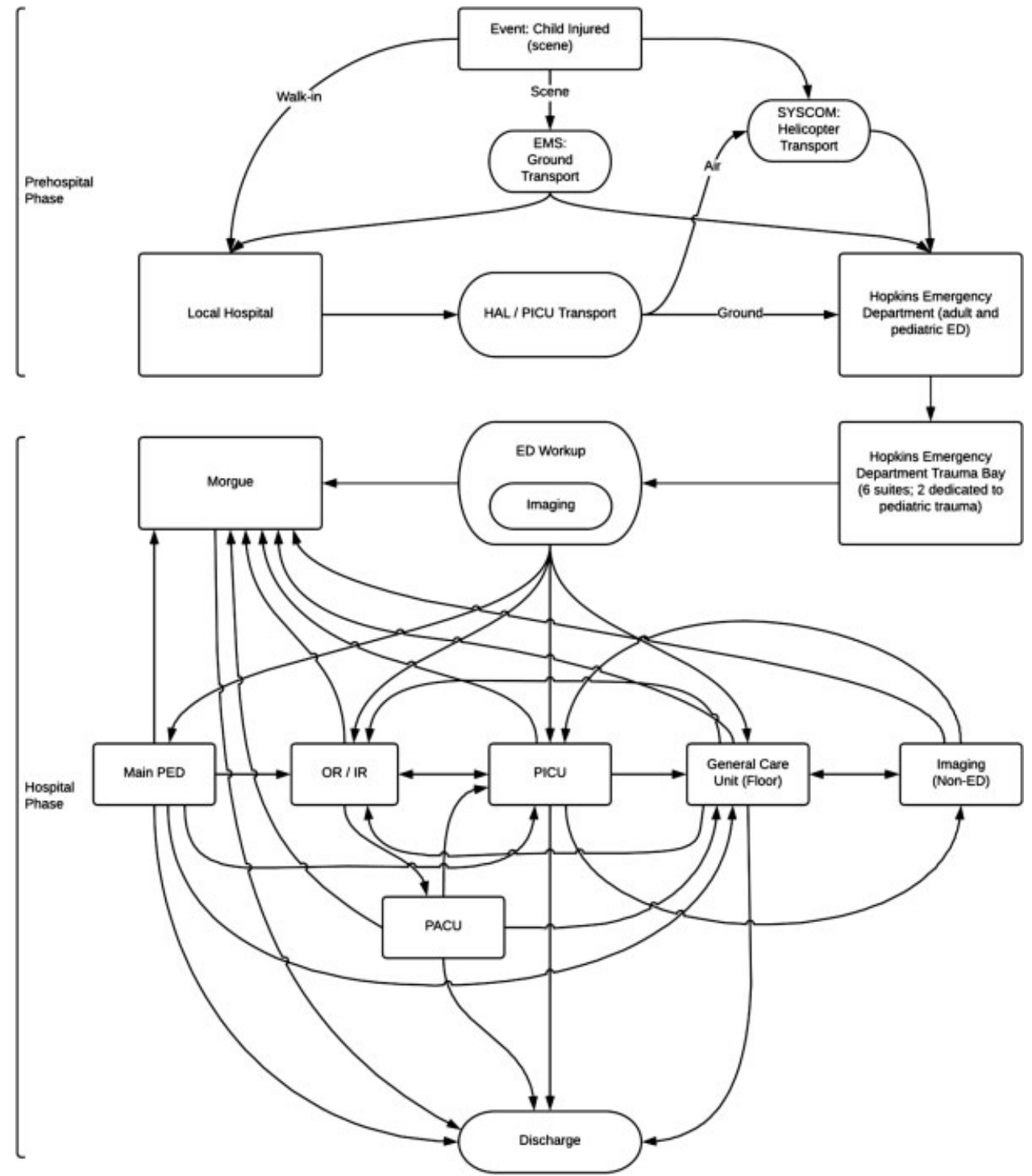

Appendix A Process map.

Appendix B Breakdown of the etiology of data quality issues in anomalous patient pathways and how they were managed

\begin{tabular}{|l|l|l|}
\hline Etiology & $\begin{array}{l}\text { Number of } \\
\text { records } \\
\text { affected }\end{array}$ & Management \\
\hline Inaccurate day & 18 & Fixed and included \\
\hline Inaccurate year & 2 & Fixed and included \\
\hline Inaccurate year & 1 & Fixed and included \\
\hline $\begin{array}{l}\text { Missing discharge } \\
\text { timestamp }\end{array}$ & 1 & Fixed and included \\
\hline $\begin{array}{l}\text { Missing encounter } \\
\text { outcome }\end{array}$ & 2 & Excluded \\
\hline $\begin{array}{l}\text { Invalid OR } \\
\text { disposition }\end{array}$ & 17 & Excluded \\
\hline Indeterminable & 3 & Excluded \\
\hline
\end{tabular}

Abbreviation: OR, operating room. 
Appendix C List of all 28 patient pathways identified showing the number of encounters for each patient pathway

\begin{tabular}{|c|c|c|}
\hline Rank & Patient pathway & $\begin{array}{l}\text { Number of } \\
\text { encounters }\end{array}$ \\
\hline 1 & Origin $\rightarrow$ ED $\rightarrow$ discharged & 877 \\
\hline 2 & Origin $\rightarrow$ ED $\rightarrow$ floor $\rightarrow$ discharged & 582 \\
\hline 3 & $\begin{array}{l}\text { Origin } \rightarrow \text { ED } \rightarrow \text { ICU } \rightarrow \text { floor } \rightarrow \\
\text { discharged }\end{array}$ & 149 \\
\hline 4 & Origin $\rightarrow \mathrm{ED} \rightarrow \mathrm{ICU} \rightarrow$ discharged & 109 \\
\hline 5 & $\begin{array}{l}\text { Origin } \rightarrow \text { ED } \rightarrow \text { floor } \rightarrow \text { OR } \rightarrow \text { PACU } \rightarrow \\
\text { discharged }\end{array}$ & 70 \\
\hline 6 & $\begin{array}{l}\text { Origin } \rightarrow \text { ED } \rightarrow \text { OR } \rightarrow \text { PACU } \rightarrow \text { floor } \rightarrow \\
\text { discharged }\end{array}$ & 26 \\
\hline 7 & $\begin{array}{l}\text { Origin } \rightarrow \text { ED } \rightarrow \text { ICU } \rightarrow \text { OR } \rightarrow \text { PACU } \rightarrow \\
\text { floor } \rightarrow \text { discharged }\end{array}$ & 18 \\
\hline 8 & Origin $\rightarrow$ ED $\rightarrow$ died & 17 \\
\hline 9 & Origin $\rightarrow$ ED $\rightarrow$ ICU $\rightarrow$ died & 11 \\
\hline 10 & $\begin{array}{l}\text { Origin } \rightarrow \text { ED } \rightarrow \text { floor } \rightarrow \text { OR } \rightarrow \text { floor } \rightarrow \\
\text { discharged }\end{array}$ & 9 \\
\hline 11 & $\begin{array}{l}\text { Origin } \rightarrow \mathrm{ED} \rightarrow \mathrm{OR} \rightarrow \mathrm{PACU} \rightarrow \\
\text { discharged }\end{array}$ & 9 \\
\hline 12 & $\begin{array}{l}\text { Origin } \rightarrow \text { ED } \rightarrow \text { OR } \rightarrow \text { PACU } \rightarrow \text { ICU } \rightarrow \\
\text { floor } \rightarrow \text { discharged }\end{array}$ & 8 \\
\hline 13 & $\begin{array}{l}\text { Origin } \rightarrow \mathrm{ED} \rightarrow \mathrm{ICU} \rightarrow \mathrm{OR} \rightarrow \mathrm{ICU} \rightarrow \text { floor } \\
\rightarrow \text { discharged }\end{array}$ & 8 \\
\hline 14 & $\begin{array}{l}\text { Origin } \rightarrow \text { ED } \rightarrow \text { OR } \rightarrow \text { ICU } \rightarrow \text { floor } \rightarrow \\
\text { discharged }\end{array}$ & 8 \\
\hline 15 & $\begin{array}{l}\text { Origin } \rightarrow \text { ED } \rightarrow \text { ICU } \rightarrow \text { floor } \rightarrow \text { OR } \rightarrow \\
\text { PACU } \rightarrow \text { discharged }\end{array}$ & 7 \\
\hline 16 & $\begin{array}{l}\text { Origin } \rightarrow \text { ED } \rightarrow \mathrm{ICU} \rightarrow \mathrm{OR} \rightarrow \mathrm{PACU} \rightarrow \\
\text { discharged }\end{array}$ & 7 \\
\hline 17 & $\begin{array}{l}\text { Origin } \rightarrow \text { ED } \rightarrow \text { OR } \rightarrow \text { Floor } \rightarrow \\
\text { discharged }\end{array}$ & 6 \\
\hline 18 & $\begin{array}{l}\text { Origin } \rightarrow \text { ED } \rightarrow \text { Floor } \rightarrow \text { ICU } \rightarrow \\
\text { discharged }\end{array}$ & 4 \\
\hline 19 & Origin $\rightarrow \mathrm{ED} \rightarrow \mathrm{OR} \rightarrow \mathrm{ICU} \rightarrow$ discharged & 3 \\
\hline 20 & Origin $\rightarrow$ ED $\rightarrow$ OR $\rightarrow$ died & 3 \\
\hline 21 & $\begin{array}{l}\text { Origin } \rightarrow \mathrm{ED} \rightarrow \mathrm{ICU} \rightarrow \mathrm{OR} \rightarrow \mathrm{ICU} \rightarrow \\
\text { discharged }\end{array}$ & 2 \\
\hline 22 & Origin $\rightarrow \mathrm{ED} \rightarrow \mathrm{OR} \rightarrow \mathrm{ICU} \rightarrow$ died & 2 \\
\hline 23 & $\begin{array}{l}\text { Origin } \rightarrow \text { ED } \rightarrow \text { OR } \rightarrow \text { PACU } \rightarrow \text { ICU } \rightarrow \\
\text { died }\end{array}$ & 1 \\
\hline 24 & Origin $\rightarrow \mathrm{ED} \rightarrow \mathrm{ICU} \rightarrow \mathrm{OR} \rightarrow \mathrm{ICU} \rightarrow$ died & 1 \\
\hline 25 & Origin $\rightarrow \mathrm{ED} \rightarrow \mathrm{ICU} \rightarrow \mathrm{OR} \rightarrow$ died & 1 \\
\hline 26 & $\begin{array}{l}\text { Origin } \rightarrow \text { ED } \rightarrow \text { floor } \rightarrow \text { OR } \rightarrow \text { PACU } \rightarrow \\
\mathrm{ICU} \rightarrow \text { discharged }\end{array}$ & 1 \\
\hline 27 & $\begin{array}{l}\text { Origin } \rightarrow \text { ED } \rightarrow \text { OR } \rightarrow \text { floor } \rightarrow \text { ICU } \rightarrow \\
\text { discharged }\end{array}$ & 1 \\
\hline 28 & $\begin{array}{l}\text { Origin } \rightarrow \text { ED } \rightarrow \text { ICU } \rightarrow \text { OR } \rightarrow \text { floor } \rightarrow \\
\text { discharged }\end{array}$ & 1 \\
\hline
\end{tabular}

Abbreviations: ED, emergency department; OR, operating room; ICU, intensive care unit; PACU, postanesthesia care unit.
Appendix D List of the 20 care transitions identified showing the number of encounters for each care transition

\begin{tabular}{|c|c|c|}
\hline Rank & Care transition & $\begin{array}{l}\text { Number of } \\
\text { encounters }\end{array}$ \\
\hline 1 & Origin $\rightarrow$ ED & 1,941 \\
\hline 2 & $\mathrm{ED} \rightarrow$ discharged & 877 \\
\hline 3 & Floor $\rightarrow$ discharged & 815 \\
\hline 4 & ED $\rightarrow$ floor & 666 \\
\hline 5 & $\mathrm{ED} \rightarrow \mathrm{ICU}$ & 314 \\
\hline 6 & ICU $\rightarrow$ floor & 180 \\
\hline 7 & $\mathrm{OR} \rightarrow \mathrm{PACU}$ & 147 \\
\hline 8 & $\mathrm{ICU} \rightarrow$ discharged & 120 \\
\hline 9 & PACU $\rightarrow$ discharged & 93 \\
\hline 10 & Floor $\rightarrow$ OR & 87 \\
\hline 11 & $\mathrm{ED} \rightarrow \mathrm{OR}$ & 67 \\
\hline 12 & PACU $\rightarrow$ floor & 44 \\
\hline 13 & $\mathrm{ICU} \rightarrow \mathrm{OR}$ & 38 \\
\hline 14 & $\mathrm{OR} \rightarrow \mathrm{ICU}$ & 24 \\
\hline 15 & $\mathrm{OR} \rightarrow$ floor & 17 \\
\hline 16 & $\mathrm{ED} \rightarrow$ died & 17 \\
\hline 17 & ICU $\rightarrow$ died & 15 \\
\hline 18 & $\mathrm{PACU} \rightarrow \mathrm{ICU}$ & 10 \\
\hline 19 & Floor $\rightarrow$ ICU & 5 \\
\hline 20 & OR $\rightarrow$ died & 4 \\
\hline
\end{tabular}

Abbreviations: ED, emergency department; OR, operating room; ICU, intensive care unit; PACU, postanesthesia care unit. 